\title{
LOS FACTORES REGIONALES DE INNOVACIÓN COMO PARTE DEL SISTEMA ECONÓMICO EUROPEO 2000 - 2010
}

\author{
REGIONAL INNOVATION FACTORS AS PART OF THE EUROPEAN \\ ECONOMIC SYSTEM $2000-2010$ \\ Lucio Leo Verástegui Huanca \\ Magister en Economía Internacional y Desarrollo Económico en la Universidad Complutense de Madrid, España. \\ Email: Investigador.lverasteguicorp@gmail.com (Autor Corresponsal)
}

[Recibido: 02/05/2016 Aceptado: 14/08/2017]

\section{RESUMEN}

Este trabajo de investigación estudia los factores Regionales de Innovación Europeos dentro del entorno económico Europeo durante el periodo 2000 - 2010. Empleando una metodología novedosa y basada fundamentalmente en un robusto marco teórico y posterior trabajo empírico. La innovación dentro de una economía nacional es fundamental para el crecimiento económico y competitividad de las empresas, así como del Gobierno. En ese sentido, la teoría evolucionista acuña las bases de la innovación como un sistema interactivo basado fundamentalmente en la sinergia de los distintos componentes que lo conforman y, así mismo, determinan el esfuerzo innovador de cada sistema. En este estudio se delinea los componentes del Sistema Regional de Innovación de 191 regiones europeas a través del Análisis Factorial, a partir de una recopilación, tratamiento y análisis exhaustivo de indicadores relacionados a la Innovación. Este trabajo puede ser usado para dar pie a futuros análisis tanto de eficiencia, mejora potencial o simplemente generar estrategias públicas eficaces.

\section{PALABRAS CLAVE}

Innovación, sistema regional, análisis factorial

\begin{abstract}
This research studies the European Regional Innovation Factors within the European economic environment during the period 2000 - 2010. We used a novel methodology and primarily based on a robust theoretical framework and subsequent empirical work. Innovation within a national economy is essential for the economic growth and competitiveness of companies, as well as the Government. In this sense, evolutionary theory establishes the bases of innovation as an interactive system based fundamentally on the synergy of the different components conforming it and, likewise, determines the innovative effort of each system. This study delineates the components of the Regional Innovation System of 191 European regions through Factorial Analysis, based on a compilation, treatment and exhaustive analysis of indicators related to Innovation. This work can lead to future analyzes of efficiency, potential improvement or simply to generate effective public strategies.
\end{abstract}

\section{KEYWORDS}

Innovation, regional system, factor analysis.

Como Citar: Verástegui, L. (2017). Los factores regionales de innovación como parte del sistema económico europeo 2000 - 2010. Quipukamayoc, 25(49), 111-119. doi: http://dx.doi.org/10.15381/quipu.v25i49.14286 


\section{INTRODUCCIÓN}

Autores como Shumpeter (1939), Solow (1956) y Freeman (1987) reconocen que la innovación es pieza fundamental en la competitividad, crecimiento económico nacional y empleo; constituye un aspecto esencial en la capacidad competitiva y de supervivencia de las empresas (Cooke, Boeholt, \& Tödtling, 2000), garantizando el posicionamiento a nivel internacional.

La Unión Europea destaca que la innovación genera empleos, construye una sociedad más ecológica, mejora la calidad de vida de sus habitantes, crea mayor competitividad comunitaria. Dutta, Escalona, Bernard, Lanvin \& Sacha (2014) refieren que hay 143 economías a nivel mundial y entre los países más innovadores se encuentra Finlandia, Reino Unido, Suiza, Suecia, Holanda, EEUU y Hong Kong, en cuanto recursos (inversión pública, infraestructura, etc.) son destinados a la Innovación.

Ahora bien, Shumpeter (1939) ha descrito la innovación como: un proceso de destrucción creativa por el cual nuevas tecnologías destruyen a las anteriores y estas últimas son más útiles; la ganancia percápita de los países industrializados no se atribuye al incremento de su población sino al progreso tecnológico junto al entorno de negocios y, por último, la expectativa de un monopolio propicia la innovación tecnológica (Scherer, 1986). Estos conceptos reflejan que la innovación surge a partir de factores externos de interrelación y que pone en marcha un esfuerzo innovador que se ve reflejado en nuevos productos y servicios. La innovación genera avances tecnológicos, pueden ser radicales o innovaciones totalmente nuevas (por ejemplo el primer teléfono mó- vil), e incrementales que son pequeñas mejoras en productos (móviles con mp3). No existe límite a las invenciones o ideas novedosas, existen nuevas combinaciones de otras innovaciones o conocimientos existentes. Para Shumpeter (1939) la innovación es el centro neurálgico del proceso de desarrollo económico y que se encuentra impulsado por empresarios. Este planteamiento ha sido heredado por la corriente evolucionista del pensamiento económico, la tecnología es un conjunto de conocimientos expresos y tácitos que residen dentro de las organizaciones las cuales participan en su creación y utilización. Esa evolución se basa en los siguientes componentes: la creación de nuevas tecnologías u organizaciones; los mecanismos que introducen novedades en el sistema (diversidad); existencia de mecanismos que seleccionan las entidades innovadoras del sistema (reduciendo la diversidad). En efecto, los recursos existentes de un sistema de innovación son el punto de partida que marcan las posibilidades y el camino a seguir, mientras que la aleatoriedad y la larga duración en el tiempo de muchos procesos de innovación sugieren que la teoría evolucionista es una aproximación más real al estudio de la innovación.

Según la teoría evolucionista, el crecimiento económico es un proceso dinámico y evolutivo basado en la adaptación, reacción y selección dentro de un sistema donde los distintos componentes son interdependientes, extrapolando este concepto a la rama de la biología - cuya esencia por sí misma es la constante evolución - tendríamos que: (1) El punto de partida es la existencia y reproducción (en la biología) y la creación de tecnologías y organizaciones (en la economía). (2) Existen mecanismos que introducen novedades en el sistema ampliando su diversificación (el desarrollo de investigaciones generan innovaciones en la economía) y las mutaciones en el contexto de la biología. (3) Existen mecanismos de selección que reduce la diversidad (en la economía esa reducción se pone de manifiesto por la elección del mercado basado en la competencia) y la selección natural para la biología (Edquist, 1997).

La innovación es clave en este sistema, siendo una actividad acumulativa, siendo el motor que convierte el sistema económico en un régimen dinámico. Este proceso evolutivo se basa en cambios endógenos - tanto debido a alteraciones bruscas como graduales - que a su vez genera un proceso continuo de adaptación o aprendizaje y selección, donde sobreviven aquellos agentes que mejor se adaptan a estos cambios.

La teoría evolucionista es interactiva e implica cambios en la gestión tecnológica de las empresas, así como, en la política tecnológica del sector público, basado principalmente en una idea de interacción continua entre distintos actores y elementos durante el proceso de innovación. Incluso después de obtener el resultado innovador este proceso interactivo sigue mediante el perfeccionamiento y diversificación de los productos y proceso, multiplicando así su efecto innovador.

Ahora bien, el proceso innovador se desarrolla dentro de un entorno en donde interactúan distintos tipos de organizaciones y agentes que se ven condicionados de alguna manera por la configuración del marco institucional en donde se desenvuelven. Este entorno es delimitado desde una perspectiva territorial - que para fines de este estudio hemos tomado las regiones europeas - ya que estas han configurado espacios activos para la 
innovación, debido a la configuración política que ha dado lugar a la aparición de nuevas formas innovadoras que son impulsadas por sus instituciones de gobierno respectivamente.

En ese sentido, el modelo interactivo señala la importancia de la estructura institucional, plasmado en el concepto de sistema nacional (regional) de innovación. Un sistema se describe como un conjunto de elementos y de relaciones entre ellos, con propiedades distintas a la suma de las partes, ejerciendo una determinada función y persiguiendo una meta específica (Nelson, 1993). Asimismo, un sistema de innovación se puede discriminar de otro, es decir, señalando sus límites y aplicando con éxito el concepto a distintos planos de análisis geográficos. Por lo tanto, que independientemente de que se trate de sistemas nacionales, regionales, locales o supranacionales todos se basan en un determinado enfoque geográfico que le atribuimos según su objeto de estudio.

Entonces, el sistema nacional/regional de innovación está conformado por: conjunto de instituciones y empresas que inciden en el cambio tecnológico y que realizan un esfuerzo organizado de investigación y desarrollo para crear y mejorar el conocimiento, se toma en cuenta también las instituciones (centros y parques tecnológicos) que ofertan servicios tecnológicos o las que ejercen relación y cooperación entre las instituciones académicas y las empresas. Además de las instituciones que favorecen la financiación de la innovación como las políticas de gobierno (Buesa \& Heijs, 2010).

Un sistema regional de innovación tiene los siguientes elementos: empresas, infraestructura pública y privada de soporte a la innovación, actuaciones públicas en relación a la innovación y el desarrollo tecnológico (incluido marco legal institucional y la política tecnológica), el marco legal (Buesa, 2002). Las características del sistema regional son:

- Es un sistema en donde interactúan distintos agentes e instituciones para la producción, difusión, empleo o para la combinación creativa de conocimientos existentes tal que la red de instituciones, del sector privado y público, cuyas actividades e interacciones inician, importan, modifican o divulgan nuevas tecnológicas (Freeman, 1987).

- El funcionamiento de un sistema regional se basa en un enfoque holístico, descrito como un proceso heterogéneo, dinámico y abierto, con retroalimentación positiva y reproducción.

- Es el resultado de un proceso histórico de causalidad acumulativa, pues la capacidad innovadora se genera a través de la acumulación de conocimientos y experiencia.

- Dentro de un sistema regional las actividades innovadoras requieren un entorno innovador donde es importante el intercambio reciproco de personal, conocimientos científicos y tecnológicos, servicios especializados e impulsos innovadores (Perrin, 1988).

El sistema regional de innovación tiene componentes que interactúan entre ellos las organizaciones e instituciones; por un lado, las organizaciones - agentes - que están conformados por las empresas, las universidades, las asociaciones de capital riesgo y las agencias públicas de política de innovación, su papel esta netamente vinculado a la producción y distribución de conocimiento. Las instituciones que reflejan las reglas de juego de las interacciones entre estos agentes, tal que se designan conjunto de prácticas, rutinas, reglas o leyes que regulan las interacciones como por ejemplo las normas de protección de propiedad intelectual o convenios universidad empresas.

Cabe señalar que el sistema no solo alberga a agentes e instituciones sino también otros factores que influyen de manera indirecta sobre las actividades innovadoras como por ejemplo las relaciones interempresariales (gasto de $\mathrm{I}+\mathrm{D}$, tamaño de empresas, nivel de internacionalización y apertura económica), estructura de mercado (condiciones de demanda, relación de cliente y proveedores), infraestructura de innovación (organismos públicos de innovación, centros de formación, parques tecnológicos, centros de transferencias tecnológicas, servicio de información y consultoría), entorno global (fuerza laboral, sistema financiero, demanda de servicios y bienes financieros, renta per cápita, cultura innovadora, descentralización política) y las relaciones de la gobernanza con la innovación (marco legal, protección de propiedad intelectual, incentivo a la innovación, promoción de la transferencia tecnológica, política internacional).

Los sistemas regionales de innovación son realidades complejas como se ha mencionado en párrafos precedentes, de tal forma que es necesario recurrir al empleo de múltiples variables para su representación empírica. En ese sentido, para trabajar con varios indicadores que reflejen la realidad y haga factible la construcción de los componentes de los Sistemas Regionales Europeos se usó una técnica estadística 
multivariable llamada Análisis Factorial, cuyo resultado obtenido -variables sintéticas llamados factores - no es más que el resumen del grueso de información contenido en los indicadores a emplear. En ese sentido, usar variables sintéticas es importante para mermar las dudas existentes respecto a que ciertas variables individuales reflejan de forma correcta las características de un sistema de innovación. Asimismo, estos indicadores resuelven problemas econométricos (multicolinealidad y falta de grado de libertad en modelos de regresión) y metodológicos (outliers o errores en las estadísticas) por sí mismos.

Un sistema de innovación contiene variedad de agentes y aspectos de su entorno que impactan directamente sobre las actividades innovadoras, de manera que un sistema regional de innovación es per se un concepto abstracto difícil de medir de forma directa en base a variables individuales. De hecho, muchos indicadores individuales reflejan conceptos parecidos y pueden ser sustituidos entre sí, pero resulta que cada uno de ellos refleja un panorama a veces distinto que el uso aislado de variables individuales marginaliza la simultaneidad o el carácter holístico del comportamiento innovador, por lo tanto, el empleo de indicadores compuestos reflejaría mejor la realidad que cada uno de ellos de forma individual.

De hecho, cada factor se basa en una media ponderada de diversas variables, lo que reduce la aparición de errores o valores atípicos de cada variable: errores en la recolección de datos, errores propios en los datos otorgados por las oficinas estadísticas y fluctuaciones ocasionadas por situaciones inesperadas. De todas formas, la creación de factores en el campo de los sistemas de innovación es un fenómeno todavía novedoso y se requiere la construcción de un consenso y la estandarización del modelo metodológico para la elaboración de índices sintéticos y la ponderación de las variables incluidas en ellos.

Ahora bien, para este estudio se han tomado la información de 191 regiones pertenecientes a 27 países de la Unión Europea bajo los criterios de disponibilidad de datos y cuestiones político administrativas similares en materia de I+D (Baumert, 2006).

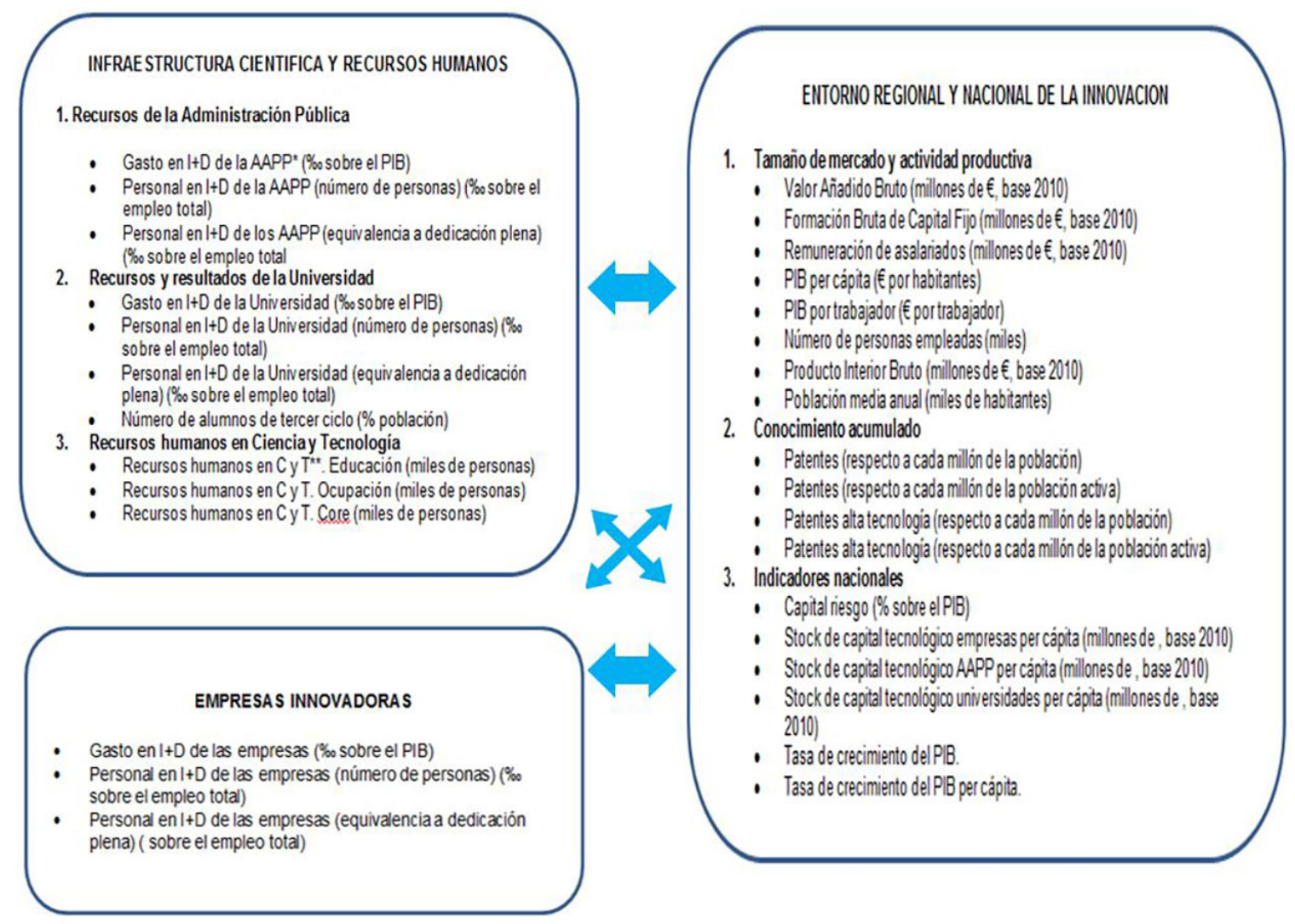

Figura 1. Variables Iniciales

Fuente: Elaboración propia a partir del esquema de Martínez (2008). 
El estudio empírico comienza con la creación de la base de datos IAIF RIS (UE), que contiene los datos relacionados a los sistemas de innovación e I+D. Se ha realizado en principio la recolección primaria de datos de EU-
ROSTAT, se ha estimado indicadores no disponibles, se ha dado el cuidado y las revisiones necesarias. Finalmente, se ha recolectado 31 variables de 191 regiones entre los años los años
2000 a 2010, ver Figura 1.

A través del programa informático SPSS se aplicó la técnica del Análisis Factorial de modo que se recoge los resultados mostrados a continuación:

\section{ENTORNO ECONOMICO REGIONAL $(31,530)$}

Número de personas empleadas (miles). $(0,973)$

Población media anual (miles de habitantes) $(0,967)$

Recursos Humanos en Ciencia y Tecnologia Ocupación (miles pers.) $(0,964)$

Recursos Humanos en Ciencia y Tecnología Core (miles pers.) $(0.956)$

Recursos Humanos en Ciencia y Tecnologia Educación (miles pers.) $(0,947)$

VAB (millones $\in$ base 2010) $(0.944)$

PIB (millones $€$ base 2010) $(0,944)$

Remuneración de asalariados (millones $€ 2010)(0,940)$

- Formación Bruta de Capital Fijo (milones $\in$ base 2010) $(0.919$

ADMINISTRACION PÚBLICA $(12,926)$
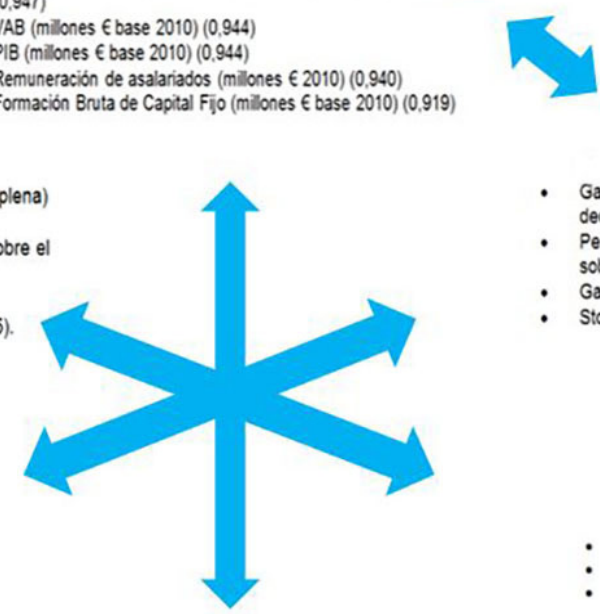

EMPRESAS INNOVADORAS $(14,830)$

Personal en I+D de las AAPP (equivalencia a dedicación plena) \%o sobre el empleo $(0.936)$.

Personal en I+D de las AAPP (número de personas) \%o sobre empleo $(0.920)$.

- Gasto en I+D de las AAPP (\%o sobre el PIB) (0.895)

- Stock capital tecnologico AAPP per cápita $(€ 2010)(0,815$.

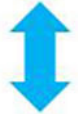

ENTORNO NACIONAL DE LA INNOVACION $(7,363)$

- Tasa de crecimiento PIB $(\%)(0.978)$

- Tasa de crecimiento PIB per cápita $(\%)(0.976)$.
- Gasto Personal en $1+D$ de las empresas (equivalencia a dedicación plena) \%o sobre el empleo $(0,909)$.

Personal en I+D de las empresas (número de personas) yo sobre el empleo $(0,902)$.

- Gasto en I+D de las empresas (so sobre el PIB) (0.897).

- Stock Capital tecnologico empresas per cápita $(€ 2010)(0.849)$.

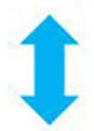

SOFISTICACION DE LA DEMANDA $(9,408)$

PIB por trabajador $(\in 2010)(0.821)$

PIB per cápita $(\in 2010)(0.732)$

Capital de riesgo (\% sobre el PIB) (por 100 para cambio de escala) $(0.569)$.

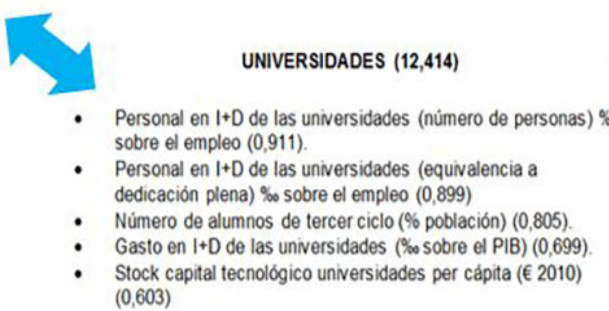

Figura 2. Resumen de Factores del Sistema Regional Europeo

Fuente: Elaboración Propia.

Los factores obtenidos representan un papel importante en los sistemas regionales de innovación:

- El factor económico regional de la innovación satura reactivos de nueve de las variables con puntuaciones altas (no menor del 0,910), este factor engloba los aspectos que inciden de manera directa sobre capacidades tecnológicas innova- doras, PIB, Formación de Capital fijo, esfuerzo humano. Pertenecientes a las 191 regiones estudiadas.

- El factor empresas innovadoras satura solo cuatro reactivos de las variables relacionadas. En ellos se recoge la capacidad de generar conocimiento y resultados tecnológicos, además de constituir centros de aprendizaje. Actúan con interés económico estimulados por la competitividad e innovación.

- El factor administración pública satura cuatro reactivos correspondientes a las variables relacionadas al personal y gasto del sector público. Este factor converge la investigación y desarrollo científico, además también gestiona y administra 
los bienes públicos llevando a cabo política social y económica.

- El factor universidad satura cinco reactivos relacionado a las respectivas variables de los cuales solo tres variables logran puntuaciones significativas por encima de 0,805 mientras que las otras dos alcanzan puntajes bajos. Además se recoge información de instituciones de enseñanza superior que fomentan la investigación científica desde el ámbito académico así como de la financiación de proyectos de investigación.

- El cuarto factor de sofisticación de la demanda satura significativamente el PIB por trabajador, PIB per cápita, capital de riesgo con puntajes de 0,$821 ; 0,732$ y 0,569 respectivamente dejando dos saturaciones irrelevantes por tener menor puntaje.

- Por último, el entorno nacional de la innovación satura la tasa de crecimiento del PIB y la tasa de crecimiento del PIB per cápita con 0,978 y 0,976 respectivamente.

Las patentes no se consideran en el análisis factorial ya que constituyen una variable output, es decir, el esfuerzo innovador de las regiones europeas está representado por el número de solicitudes de patentes que se obtienen cada año por parte de empresas, instituciones públicas y otros agentes. Garantizan un nivel mínimo de originalidad y debido a su alto coste - en tiempo y en dinero - tienen una probabilidad mayor de convertirse en innovaciones (Buesa \& Navarro, 2001). Es por eso que contamos con variables relacionadas no solo al factor esperado sino también a los otros factores. En nuestro caso contamos con 27 variables relacionadas:

- Para realizar el Análisis Factorial las correlaciones entre variables tienen que ser altas. La prueba de esfericidad de Bartlett se emplea para probar la hipótesis de que la matriz de correlaciones es una matriz identidad. En la tabla 1 se observa que el grado de significación ("sig") es de 0,000 , por lo que se rechaza la hipótesis nula. Por otro lado, observamos la medida de adecuación muestral KMO de Kaiser - Meyer - Olkin - que permite comparar magnitudes de coeficientes de correlación obtenidos con las magnitudes con coeficientes de correlación parcial, por lo tanto, cuando este índice toma un valor bajo se desaconseja la aplicación del Análisis Factorial. Kaiser elaboró una escala en donde los índices KMO entre 0,90 y 1 se catalogan como "maravillosos", entre 0,80 y 0,90 como "meritorios", entre 0,70 y 0,80 como "medianos", entre 0,60 y 0,70 como "mediocres", entre 0,50 y 0,60 como "bajos" y entre 0 y 0,50 como "inaceptables". En ese sentido, nuestro valor del KMO es de 0,826 , y supone que es adecuada para realizar la técnica.

Tabla 1.

Prueba de KMO y test de Bartlett

Fuente: Elaboración propia a partir de la aplicación estadística SPSS.

\begin{tabular}{|c|c|c|}
\hline \multicolumn{2}{|c|}{ Medida Kaiser-Meyer-Olkin de adecuación de muestreo } & 0,826 \\
\hline \multirow{3}{*}{ Prueba de esfericidad de Bartlett } & Aprox. Chi-cuadrado & 123338,948 \\
\hline & gl & 0,351 \\
\hline & Sig. & 0,000 \\
\hline
\end{tabular}

- Se denomina comunalidad a la proporción de varianza explicada por los componentes analizados. En el caso las comunalidades iniciales de las variables son siempre iguales a uno $(1,000)$. Una comunalidad cercana a 0 indica que los componentes no indican variabilidad de una variable mientras que si se acerca más a 1 la variable quedara totalmente explicada por los componentes. En el presente caso se observa en general que los 27 reactivos son explicados por componentes ya que no existen valores bajos próximos a cero.

- El método de extracción de com- ponentes principales parte de la idea de maximizar la varianza explicada, es decir, el primer componente es aquel que contribuye mejor a explicar la varianza total, el segundo aporta un máximo de la varianza residual restante (siendo independiente del primero) y sucesivos componentes explican pro- 
gresivamente pequeñas cantidades del total de la varianza. Al momento de determinar los factores, partimos de la regla de conservar aquellos componentes con autovalores mayores a la unidad, es decir, un autovalor se define como la varianza explicada por cada factor. En la tabla 2 apreciamos que existen seis componentes con autovalor mayor a cero, lo que indica que van a ser extraídos seis factores que explican el $88,472 \%$ del total de la varianza.

Tabla 2.

Varianza Total Explicada por Componentes

Fuente: Elaboración propia a partir de la aplicación estadística SPSS.

\begin{tabular}{|c|c|c|c|c|c|c|c|c|c|}
\hline $\begin{array}{l}\text { C } \\
\mathbf{o}\end{array}$ & \multicolumn{3}{|c|}{ Auto valores iniciales } & \multicolumn{3}{|c|}{$\begin{array}{c}\text { Sumas de extracción de cargas } \\
\text { al cuadrado }\end{array}$} & \multicolumn{3}{|c|}{$\begin{array}{l}\text { Sumas de rotación de cargas al } \\
\text { cuadrado }\end{array}$} \\
\hline $\begin{array}{l}\text { p } \\
\text { o } \\
\text { n } \\
\text { e } \\
\text { n } \\
\text { t } \\
\text { e }\end{array}$ & Total & $\begin{array}{c}\% \text { de } \\
\text { varianza }\end{array}$ & $\begin{array}{c}\% \\
\text { acumulado }\end{array}$ & Total & $\begin{array}{c}\% \text { de } \\
\text { varianza }\end{array}$ & $\begin{array}{c}\% \\
\text { acumulado }\end{array}$ & Total & $\begin{array}{c}\% \text { de } \\
\text { varianza }\end{array}$ & $\begin{array}{c}\% \\
\text { acumulado }\end{array}$ \\
\hline 1 & 10,912 & 40,415 & 40,415 & 10,912 & 40,415 & 40,415 & 8,513 & 31,530 & 31,530 \\
\hline 2 & 4,723 & 17,494 & 57,910 & 4,723 & 17,494 & 57,910 & 4,004 & 14,830 & 46,360 \\
\hline 3 & 3.156 & 11,687 & 69,597 & 3,156 & 11,687 & 69,597 & 3,490 & 12,926 & 59,286 \\
\hline 4 & 2,080 & 7,705 & 77,302 & 2,080 & 7,705 & 77,302 & 3,352 & 12,414 & 71,701 \\
\hline 5 & 1,882 & 6,969 & 84,271 & 1,882 & 6,969 & 84,271 & 2,540 & 9,408 & 81,108 \\
\hline 6 & 1,134 & 4,201 & 88,472 & 1,134 & 4,201 & 88,472 & 1,988 & 7,363 & 88,472 \\
\hline
\end{tabular}

- En la figura 2 se recogen los pesos factoriales de cada variable en los seis componentes extraídos. El peso factorial indica el grado de correlación entre variable y componente. Observamos que se muestran pesos mayores a 0,40 (aquello valores que acompañan al indicador entre paréntesis). También observamos que el primer componente contiene la mayor parte de reactivos relacionados entre sí. En la tabla 2 se observa la información extraída de los indicadores en cada componente y representada por el $\%$ de varianza respectivo.

- La rotación de componentes ayuda a ser sencilla la interpretación de componentes tal que en nues- tro caso empleamos el método de rotación varimax, en el cual se considera los factores como ortogonales, es decir que no están correlacionados entre sí. Entonces, esta rotación transforma la matriz factorial inicial en una rotada de fácil interpretación conformada por una combinación lineal de la primera que explica la misma cantidad de la varianza inicial solo que esta vez ordenada. Para el estudio se ha obtenido una matriz con seis componentes denominados factores y entroncando con la teoría explicada en párrafos precedentes se han denominado: Entorno económico regional de la Innovación, empresas innovadoras, Administración Pública, Universidades, so- fisticación de la demanda y Entorno Nacional de la Innovación que se resume en el figura 2.

En definitiva el Análisis Factorial es consistente y cumple con los requisitos conceptuales y técnicos que se exige, se ha reducido la información a seis factores que se utilizaran como inputs en el modelo productivo de la innovación.

\section{CONCLUSIONES}

Primero: en los años setenta la tecnología era considerada como información, cuyo proceso de producción era resultado de la actividad de instituciones de investigación (exógena al sistema económico) y de las empresas 
innovadoras. Este modelo lineal su giere que el producto (output) está altamente relacionado linealmente con el factor de entrada (input) dando como resultado la función de producción.

Esta visión del cambio tecnológico conceptualiza la $\mathrm{I}+\mathrm{D}$ como una actividad aislada, llevada a cabo en centros de investigación y que no se deja influir por incentivos desde el mercado y otras unidades de la empresa (Heijs, Buesa \& Baumert, 2007). Constituye un proceso lineal y secuencial llevado a cabo en fases aisladas que se inicia con la fase de investigación básica y finaliza con la fase de introducción de las innovaciones en el mercado. En definitiva el modelo lineal de cambio tecnológico niega de manera virtual factores como la influencia de instituciones, estrategias y actitudes competitivas de otras empresas y países o factores relacionados con la demanda y la educación y por su parte las políticas basadas en el modelo lineal están dirigidas a la creación de innovaciones mediante la creación de centros de investigación, el apoyo a la $\mathrm{I}+\mathrm{D}$ básica para tecnologías claves o la financiación directa de las actividades empresariales.
En síntesis el modelo lineal de cambio tecnológico está basado solo en las actividades tecnológicas aisladas realizadas por universidades, centros de investigación o instituciones de investigación y desarrollo (I+D) mientras que el modelo interactivo o evolucionista está basado en la interacción continua entre los distintos actores y elementos durante todo el proceso de innovación y la comercialización posterior del output hasta incluso después en términos de perfeccionamiento y diversificación.

Segundo: La construcción del modelo factorial depende de la buena elección de variables que hagan emerger los factores esperados; no hay posibilidad que se ponga de manifiesto un determinado factor si no existe ninguna variable capaz de saturarlo.

El papel de la innovación en un país es fundamental para el desarrollo y la competitividad de los agentes que participan en ese entorno. En efecto, la innovación constituye un factor importante en el crecimiento económico de los países, la creación de empleo, la competitividad y el posicionamiento internacional.
El enfoque evolucionista robustece el estudio de Sistemas Regionales de Innovación dotándole de sentido y profundidad al hecho evolutivo de la innovación y de sus relaciones entre sus agentes.

A partir de la base de datos y el Análisis Factorial aplicado se ha identificado seis factores que entroncan con los componentes principales de los sistemas Regionales de Innovación Europeos: Entorno económico regional $(31,530)$, empresas innovadoras (14,830), Administración Pública $(12,926)$, Universidades $(12,414)$, Sofisticación de la Demanda $(9,408)$ y el Entorno Nacional de la innovación (7,373).

Por último, desde el pensamiento sistémico se sugiere lograr el equilibrio de los seis factores de innovación para asegurar un alto grado de eficiencia, dinamismo económico y aprovechamiento de las ventajas resultado de su sinergia; por eso las políticas de apoyo a la innovación están llamadas a incentivar mayores esfuerzos innovadores tanto en el sector público así como privado. 


\section{REFERENCIAS BIBLIOGRÁFICAS}

Baumert, T.H. (2006). Los determinantes de la innovación. Un análisis aplicado sobre las regiones de la Unión Europea. (Tesis doctoral). Universidad Complutense de Madrid, Madrid.

Buesa, M. (2002). El sistema regional de I+D+I de la Comunidad de Madrid. Madrid: Comunidad de Madrid.

Buesa, M. \& Navarro, M. (2001). Indicadores de la ciencia, la tecnología y la innovación, metodología y fuentes para la CAPB y Navarra - Cuadernos de ciencias sociales y económicas. Madrid: Editorial Complutense.

Buesa, M. \& Heijs, J. (2010). Tecnología e Innovación - Situación Económica y Social de la Comunidad de Madrid 2009. Madrid: Universidad Complutense de Madrid.

Cooke, P.H., Boekholt, P. \& Tödtling, F. (2000). The Governance of Innovation in Europe. Regional Perspectives on Global Competitiveness. New York: Pinter.

Dutta, S., Escalona, R., Bernard, A., Lanvin, B. \& Sacha, V. (2014). The Global Innovation Index 2014 - Nurturing New Sources of Growth by Developing the Human Factor in Innovation.

Edquist, C. (1997). Systems of innovation: technologies, institutions, and organizations. London: Pinter.

Freeman, C.R. (1987). Technology and Economic Performance - Lessons from Japan. London: Printers Publishers.

Heijs, J., Buesa, M. \& Baumert, T.H. (2007). Sistemas nacionales de innovación-conceptos, perspectivas y desafíos. Madrid. Comunidad de Madrid.

Martínez, M. (2008). Tipología y Eficiencia de los Sistemas Regionales de Innovación. (Tesis doctoral). Universidad Complutense de Madrid, Madrid.
Nelson, R. (1993). National Innovation Systems - A comparative Analysis. Londres: Oxford University Press.

Perrin, J. C. (1988). A desconcentrated technology policy, Lessons from the Sophia Antipolis Experience. Environment and Planning C, Government and Policy, 6(4), $414-426$.

Scherer, F.M., (1986). Innovation and growth - Shumpeterian perspectives. Cambridge: London.

Schumpeter, J. A. (1939). Business Cycles- A Theoretical, Historical, And Statistical Analysis of The Capitalist Process. New York: McGraw- Hill.

Solow, R. (1956). A Contribution To The Theory Of Economic Growth. Quaterly Journal of Economics, 70(1), 65-94. 